\title{
Optimal terminal sliding mode control for second-order motion systems
}

\author{
Michael Ruderman
}

\begin{abstract}
Terminal sliding mode (TSM) control algorithm and its non-singular refinement have been elaborated for two decades and belong, since then, to a broader class of the finite-time controllers, which are known to be robust against the matched perturbations. While TSM manifold allows for different forms of the sliding variable, which are satisfying the $q / p$ power ratio of the measurable output state, we demonstrate that $q / p=0.5$ is the optimal one for the second-order Newton's motion dynamics with a bounded control action. The paper analyzes the time-optimal sliding surface and, based thereupon, claims the optimal TSM control for the secondorder motion systems. It is stressed that the optimal TSM control is fully inline with the Fuller's problem of optimal switching which minimizes the settling time, i.e. with timeoptimal control of an unperturbed double-integrator. Is is also shown that for the given plant characteristics, i.e. the overall inertia and control bound, there is no need for additional control parameters. The single surface design parameter might (but not necessarily need to) be used for driving system on the boundary layer of the twisting mode, or for forcing it to the robust terminal sliding mode. Additional insight is given into the finite-time convergence of TSM and robustness against the bounded perturbations. Numerical examples with different upper-bounded perturbations are demonstrated.
\end{abstract}

\section{INTRODUCTION}

Terminal sliding mode control belongs to the class of finite time controllers [1], see e.g. [2] for survey, for which a finitetime convergence can be guaranteed. The finite-time convergence is here valid for both, the reaching phase during which the state trajectories reach the predefined sliding surface, and the sliding phase of a convergence to the state equilibrium. In other words, when the control task is formulated as a zeroreference and non-zero initial value problem, the controlled system attains the origin in the finite-time. Yet, for the reaching phase one refers to a finite-time convergence of the sliding variable to zero, while for the second (sliding) phase a finite-time convergence of the output tracking error to zero is meant. For more details on the reaching and sliding phases and convergence of the sliding mode control systems we refer to the seminal literature e.g. [3], [4], [5].

Based on [6], [7] the so-called terminal sliding mode control has been proposed in [8] and, later, brought into a non-singular form in [9]. The difference between both will be summarized in the preliminaries provided in Section $\Pi$ Remarkable is a fact that for the second-order systems, which are in focus of this work, the terminal sliding mode surface, correspondingly control, coincides with the Fuller's problem of optimal relay switching [10]. The latter has also been

This work has received funding from the EUs H2020-MSCA-RISE research and innovation programme under grant agreement No 734832 .

M. Ruderman is with Faculty of Engineering and Science, University of Agder, Norway. Email: michael.ruderman@uia.no brought into context and analyzed in terms of designing highorder sliding mode control algorithms in [11]. It should be recalled that for the second-order Newtonian dynamics of a relative motion, it is always possible to bring the system to an equilibrium in finite time, by using a bounded control, i.e. through inherently bounded actuation forces. The wellknown approach to this problem leads to the co-called bangbang control [12], which is the time-optimal solution of an unperturbed double-integrator control problem. We will also briefly sketch it in Section $\amalg$ for convenience of the reader.

In the rest of the paper, we will show that an optimal terminal sliding mode control can be designed for motion systems with the bounded perturbations and control actions, based on the time-optimal switching of double-integrator. The main results with analysis are given in Section IV Section $\nabla$ demonstrates three numerical examples of the control performance for different-type matched perturbations. The main conclusions are briefly drawn in Section VI

\section{PRELIMINARIES}

The so-called terminal sliding mode control suggests the first-order terminal sliding variable, cf. [9],

$$
s=x_{2}+\beta x_{1}^{q / p},
$$

where $\beta>0$ is a design parameter and $p>q$ are the positive odd constants [9]. Recall that the terminal sliding mode controllers belong to the class of second-order sliding modes with finite-time convergence, see e.g. [13]. For a terminal sliding mode, the control of a dynamic system $\ddot{x}_{1}=\dot{x}_{2}=u$, in the most simple case, is given by

$$
u=-\alpha \operatorname{sign}(s),
$$

where $\alpha>0$ is the control parameter. The requirement on $p, q$ to be odd [9] can be relaxed, and a frequently encountered terminal sliding surface is written as, cf. [13],

$$
s=x_{2}+\beta \sqrt{\left|x_{1}\right|} \operatorname{sign}\left(x_{1}\right) .
$$

By taking the time derivative of the sliding surface one can show that, cf. [14],

$$
\dot{s}=-\alpha \operatorname{sign}(s)+\beta x_{2}\left(2 \sqrt{\left|x_{1}\right|}\right)^{-1} .
$$

One can recognize that a singularity occurs if $x_{2} \neq 0$ and $x_{1}=0$. This situation does not occur in an ideal sliding mode, i.e. $s=0$. However, the terminal sliding mode control with surface (1) cannot guarantee an always bounded control signal before the system is on the sliding manifold $s=0$, i.e. during the reaching phase which depends on the 
initial conditions. Once on the surface, the sliding variable dynamics (4) reduces to

$$
2 \dot{s}=-2 \alpha \operatorname{sign}(s)-\beta^{2} \operatorname{sign}\left(x_{1}\right) .
$$

Thereupon, one can show that the existence condition $s \dot{s}<$ 0 of an ideal sliding mode is fulfilled for $\beta^{2}<2 \alpha$. For that case, the trajectories of the system reach the surface (3) and remain there for all times afterwards. This control system behavior is often denoted as a terminal mode, cf. [15], [14]. When the design parameters are assigned as $\beta^{2}>2 \alpha$, the trajectories of the system do not remain on the sliding surface, while the control still switches at $s=0$, according to (2), (3). That case the control system trajectories proceed in the so-called twisting mode, cf. [15], [14].

For overcoming the singularity of the sliding surface (1), the non-singular terminal sliding mode control with

$$
s=x_{1}+\beta^{-1} x_{2}^{p / q},
$$

has been proposed in [9], while $\beta, p$, and $q$ parameters are kept the same as before. This control works around the surface singularity problem while, at the same time, (1) and (6) describe one and the same switching surface, seen from the geometrical interpretation, in the $\left(x_{1}, x_{2}\right)$ coordinates.

\section{TIME OPTIMAL CONTROL OF SECOND-ORDER MOTION SYSTEMS}

We consider a generalized 1 second-order motion system

$$
\ddot{x}(t)=m^{-1} u(t)
$$

of an inertial body $m$, which is first not affected by any counteracting, correspondingly disturbing, forces. For the sake of simplicity and without loss of generality we focus on the 1DOF motion, so that the scalar state variables of motion are $x_{1}=x$ and $x_{2}=\dot{x}$. The control input force, here and for rest of the paper, is constrained as $u \in[-U,+U]$. Here it is worth emphasizing that the bounded (often denoted as saturated) control signal, correspondingly actuation force, is in focus of our optimal terminal sliding mode control. Consequently, $U$ will appear as a system parameter in the following analysis and control synthesis.

Assuming an initial state $\left[x_{1}(0), x_{2}(0)\right]^{T}$ and a final state $\left[x_{1}\left(t_{f}\right), x_{2}\left(t_{f}\right)\right]^{T}$ the time-optimal control [10], [12], [16] will minimize the cost criterion

$$
J(u)=t_{f}=\int_{0}^{t_{f}} d t,
$$

thus minimizing the overall convergence (or settling [10]) time $t_{f}$. Following the Pontryagin's minimum principle and solving the constrained (by control limit $U$ and initial and final conditions) optimization problem (8) leads to the Hamiltonian function

$$
H=\lambda_{0}+\lambda_{1}(t) x_{2}(t)+\lambda_{2}(t) u(t)
$$

\footnotetext{
${ }^{1}$ We mean here the generalized coordinates $x$ and forces $u$ for not explicitly distinguishing between the translational and rotational degrees of freedom (DOFs). As consequence, $m$ is used for denoting equally both quantities, inertial mass and moment of inertia respectively.
}

with $\lambda_{0-2}$ to be the vector of Lagrange multipliers. Minimizing the Hamiltonian function yields the time-optimal control of the form, cf. with [16],

$$
u^{o}(t)= \begin{cases}+U, & \text { for } \lambda_{2}^{o}(t)<0 \\ 0, & \text { for } \lambda_{2}^{o}(t)=0 \\ -U, & \text { for } \lambda_{2}^{o}(t)>0\end{cases}
$$

where $\lambda_{2}^{o}(t)$ is the control-related optimal Lagrange multiplier. Substituting this control law into $x_{2}$-dynamics results in a two-point boundary value problem, see [16] for details, from which the solution of optimal Lagrange multiplier gives

$$
\lambda_{2}^{o}(t)=-c_{1}^{o} t+c_{2}^{o} .
$$

Finally, the optimal constant values $\left(c_{1}^{o}, c_{2}^{o}\right) \neq(0,0)$ need to be found such that the two-point boundary value problem is solved. With closer look on (10), (11) one can recognize that: (i) the motion system (7) is always fully either accelerated or decelerated, (ii) the calculated multiplier (11) determines the time instant of exactly one switching between both control actions, e.g. from $+U$ to $-U$ when $x_{1}(0)<x_{1}\left(t_{f}\right)$. Such control behavior is well known as the so-called "bang-bang" control, while the optimal switching time, resulting from 111, is always depending on the initial and final states. Here it is worth recalling that the time-optimal control (10), solved in terms of a switching time instant 111, was formulated as the Fuller's problem in [10] and further analyzed for an optimal sliding mode control in [11], in both cases in terms of the state variables, correspondingly output tracking error.

For the controlled system (7), (10) we are next to analyze the decelerating trajectory, i.e. the state trajectory after the time-optimal switching at $t=\tau$, and that for $u(t)=$ $-U \forall \tau \leq t \leq t_{f}$. For the sake of simplicity and without loss of generality we will assume $x_{1}\left(t_{f}\right)=x_{2}\left(t_{f}\right)=0$, that for the rest of the paper, meaning zero equilibrium point. Consequently, all our following analysis and developments reduce to the problem of convergence to a stable equilibrium in the origin, while other control tasks with $\left(x_{1}\left(t_{f}\right), x_{2}\left(t_{f}\right)\right) \neq 0$ can be converted to that one by an appropriate transformation of the state coordinates. For the phase-plane analysis, the system (7) with $u=-U$ can be rewritten as

$$
\dot{x} d \dot{x}=-U m^{-1} d x,
$$

and after integration of both sides and substitution of $x_{1}$ and $x_{2}$ one obtains the parabolic trajectory

$$
x_{1}=-0.5 m U^{-1} x_{2}^{2} .
$$

Obviously, the upper branch of the $\left(x_{1}, x_{2}\right)$-parabola con-

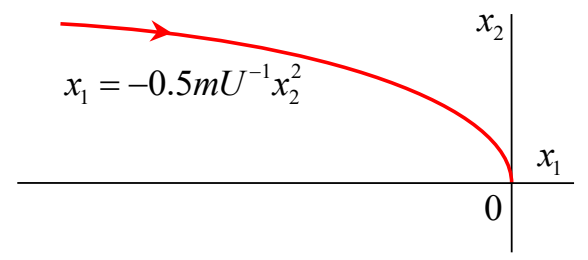

Fig. 1. Decelerating trajectory of time optimal control 10 . 
stitutes the motion trajectory until $t=t_{f}$. The motion trajectory is steered with the maximal possible deceleration forced by $u(t)=-U$. An example of the converging state trajectory, driven by the time-optimal control (10) during the decelerating phase, is shown in Fig. 1

\section{OPTIMAL TERMINAL SLIDING MODE CONTROL}

Now, with the preliminaries from Section I and time optimal control summarized in Section III we are in the position to formulate the optimal terminal sliding mode control for the perturbed second-order motion systems.

The proposed optimal terminal sliding surface is given by

$$
s=x_{1}+\alpha m U^{-1} x_{2}^{2} \operatorname{sign}\left(x_{2}\right),
$$

with $\alpha>0$ to be the single (optional) design parameter. Note that the proposed switching, correspondingly sliding, surface (13), cf. [10, eq. (53)], has the same form as the non-singular terminal sliding mode algorithm, see [14], with $q / p=0.5$ rate cf. Section Important to notice is that both surface shaping factors, $U$ and $m$, do not represent any free parameters to be tuned. They are rather the inherent physical quantities which are characterizing the motion system plant (7). One can recognize that the trajectory (12) (of a timeoptimal controlled system) coincides with the sliding surface (13) for $\alpha=0.5$. Further we will show that for that case, the boundary layer of the so-called twisting mode appears, cf. [15], [14]. The control value is

$$
u(s)=-U \operatorname{sign}(s),
$$

in which the control gain factor is the maximal possible one, i.e. inherently limited by the constrained actuator force.

The sufficient condition for existence of the terminal sliding mode on the surface (13) can be shown by assuming the candidate Lyapunov function

$$
V=0.5 s^{2}
$$

for which

$$
0.5 \frac{d}{d t} s^{2}<0
$$

is, respectively, required. That means for the trajectory which has once reached and crossed the surface $s=0$ at $t=t_{r}$, it remains on it $\forall t>t_{r}$. The corresponding motion along $s=0$, denoted as terminal mode, requires $s \dot{s}<0$ to be always fulfilled. For showing it, we exemplary consider $x_{2}>0$, thus focusing, yet without loss of generality, on the upper parabolic branch in the second quadrant of the phase-plane only. Next we summarize $\alpha m U^{-1}=: C$, for the sake of simplicity, and with these simplifications in mind the terminal sliding surface 13 can be written as a parabolic equation $s=x_{1}+C x_{2}^{2}$. Taking the time derivative and substituting the control (14) into the dynamics (7) one obtains

$$
\dot{s}=x_{2}-2 m^{-1} C U x_{2} \operatorname{sign}(s) .
$$

Multiplying both sides of 17 with $s$, the existence condition (16) results in

$$
x_{2} s-2 m^{-1} C U x_{2}|s|<0 .
$$

Substituting back the plant parameters, instead of $C$, and solving the inequality 18 with respect to the single free factor $\alpha$, the existence condition of the optimal terminal sliding mode results in

$$
\alpha>0.5 \text {. }
$$

Important to notice is that if the design parameter is selected as $0<\alpha \leq 0.5$, the existence condition (16) of the sliding mode becomes violated, and the trajectories do not remain on $s=0$ upon crossing. Nevertheless, the manifold (13) appears further on as a switching surface, and the trajectories will reach asymptotically the origin according to (7) and (14). This convergence behavior appears as a twisting mode, in which the frequency of switching (upon crossing the surface (13) with $0<\alpha \leq 0.5$ ) increases towards infinity as the trajectory approaches the origin. Recall that the trajectory converges towards origin while circulating around it. The convergence of the twisting mode can be directly proved geometrically, since $\left\|x_{1}, x_{2}\right\|\left(t_{s 2}\right)<\left\|x_{1}, x_{2}\right\|\left(t_{s 1}\right)$ for all pairs of two consecutive switching (correspondingly $s$-crossing) at $t_{s 2}>t_{s 1}$. This is valid as long as $\alpha>0$. When $\alpha \rightarrow 0$, the switching surface $s \rightarrow x_{1}$ and the twisting occurs without convergence, while representing the boundary case of a harmonic oscillator. The latter is nothing but the double-integrator with a zero-crossing relay in feedback. The stability of the twisting mode has also been explicitly addressed in [15] by means of the Lyapunov's method. It is also worth noting that for $0.25 \leq \alpha \leq 0.5$, the sliding surface still falls into class of the Fuller's problem, cf. [10], [11]. Also one can easily recognize that the positive $\alpha$ appears, from a plant parameters viewpoint, as a gain factor which captures the multiplicative uncertainties in $m$ and $U$, cf. 13.

The phase-portrait with trajectories of the system (7), (14) is exemplary shown in Fig. 2 together with the corresponding surface (13), that for the different $\alpha$-values. Here

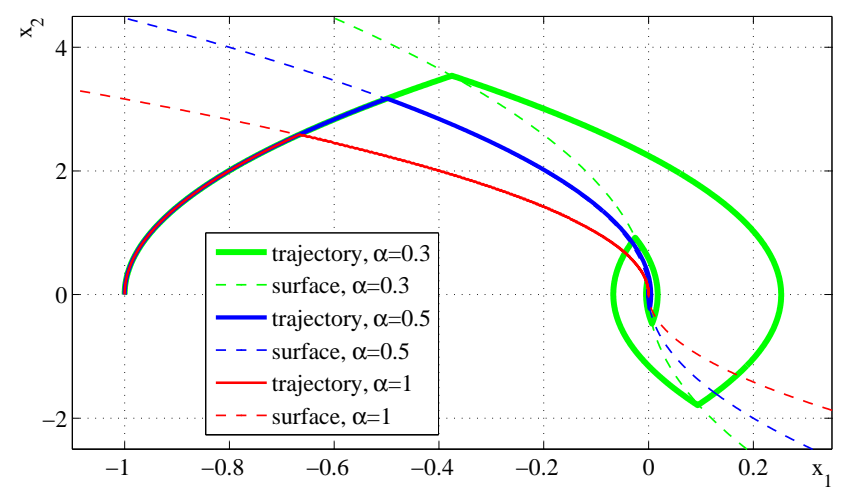

Fig. 2. Sliding surface and trajectories for different $\alpha$ values.

the system parameters are assigned as in the numerical examples provided in Section [V] while the initial values $\left(x_{1}\left(t_{0}\right), x_{2}\left(t_{0}\right)\right)=(-1,0)$ are assumed for all three $\alpha$ values. One can directly notice that once the switching surface is reached, the control with $\alpha=0.3$, which violates [19, does not stay sliding on the surface, and the trajectory 
converges in a twisting mode. Note that here the chattering, due to non-ideal switching and numerical computational issues, does not appear until the states reach the origin, meaning until $\left(x_{1}\left(t_{f}\right), x_{2}\left(t_{f}\right)\right) \rightarrow \mathbf{0}$.

In order to establish the reachability condition, see [17], [5], one consider the candidate Lyapunov function (15) for which the following conditions should be satisfied: (i) $V(s)$ is positive definite $\forall s$, (ii) $\dot{V}<0$ for $s \neq 0$. For showing the finite-time convergence and, therefore, global stability the so-called $\eta$-reachability [17] condition

$$
s \dot{s}<-\eta|s|
$$

can be evaluated, in which $\eta>0$ is a small constant which ensures the reachability condition is satisfied. One slightly modifies the above condition (ii) to be

$$
\dot{V} \leq-\gamma \sqrt{V}, \quad \gamma=\sqrt{2} \eta
$$

Integrating both sides of inequality 21 one obtains, cf. [5],

$$
\sqrt{V}(t) \leq-0.5 \gamma t+\sqrt{V(0)}
$$

Since reaching the sliding surface at finite-time $t_{r}$ means $V\left(t_{r}\right)=0$, one can obtain, out from (22), the following

$$
t_{r} \leq \gamma^{-1} 2 \sqrt{V(0)}=\gamma^{-1} 2 \sqrt{0.5}|s(0)| .
$$

One can recognize that a bounded finite time can be guaranteed by (23) and it depends on the initial value of the sliding manifold only. In order to evaluate the $\eta$-reachability condition for the control system (7), 13), (14), we first rewrite 20) as

$$
\operatorname{sign}(s) \dot{s}<-\eta \text {. }
$$

Taking the time derivative of 13 and substituting the control system dynamics (7), 14, one obtains

$$
\dot{s}=x_{2}-2 \alpha\left|x_{2}\right| \operatorname{sign}(s) .
$$

Combining (24) and 25 and separating the variables we achieve the $\eta$-reachability condition as

$$
x_{2} \operatorname{sign}(s)<-\eta+2 \alpha\left|x_{2}\right| .
$$

One can see that it is always possible to find a small positive constant $\eta$ such that the condition (26) is fulfilled $\forall x_{2}$.

Once the existence and reachability conditions, 19) and (26) respectively, are satisfied it is indicative to analyze the control system robustness against the perturbations. The external and internal perturbations matched by the control will directly affect the right-hand side of (7) and, in worst case, produce a counteracting (disturbing) force during the reaching phase, for which $|u|(t)=U$ during the time $0<$ $t<t_{r}$. On the other hand, the sudden 'fast' perturbations, once they are occurring during the sliding mode, will diverge the trajectories from the sliding manifold, thus giving rise (again) to the reachability problem. Such transient loss of the sliding mode leads, in turn, to $u(t)=$ const $\in\{-U, U\}$

\footnotetext{
${ }^{2}$ Here we refer to the internal perturbations as well, since these may arise from the plant uncertainties and internal dynamics which are not captured by the motion system in form of the double-integrator 7.
}

during the time $t_{d}<t<t_{r}$, where $t_{d}>0$ is the last instant of losing the sliding manifold $s$ due to appearance of the perturbations. In order to guarantee that the optimal terminal sliding mode control can compensate for the unknown perturbations $\xi(t)$, the single boundedness condition

$$
|\xi|<U
$$

is required for all times $t>0$ of the control operation.

In the following, we will demonstrate different examples of the optimal terminal sliding mode control applied to the perturbed motion system. For that purpose, the system plant is extended by the matched upper bounded perturbation (27), thus resulting in the modified (7) which becomes

$$
\ddot{x}(t)=m^{-1}(u(t)+\xi(t)) .
$$

\section{NUMERICAL EXAMPLES}

The assumed numerical parameters, here and for the rest of the paper, are $m=0.1, U=1, \alpha=0.6$. All the controlled motion trajectories start at the initial state $\left(x_{1}\left(t_{0}\right), x_{2}\left(t_{0}\right)\right)=$ $(-1,0)$, while the control set point is placed in the origin, meaning $\left(x_{1}\left(t_{f}\right), x_{2}\left(t_{f}\right)\right)_{\text {ref }}=\mathbf{0}$. The implemented and executed simulations are with $1 \mathrm{kHz}$ sampling rate and the (most simple) first-order Euler numerical solver.

\section{A. Motion system with nonlinear friction}

The motion system dynamics (7) is first considered as being affected by the nonlinear Coulomb friction $f\left(x_{2}, t\right)$ which, moreover, includes the continuous presliding transitions, see [18] for details. It is worth emphasizing that the modeled Coulomb friction force is not discontinuous at zero velocity crossing, while a saturated friction force at steadystate results in $F_{c} \operatorname{sign}(\dot{x})$, where $F_{c}>0$ is the Coulomb friction constant. The friction force, which appears as an internal perturbation $\xi=-f$, is assumed to be bounded by the half of the control amplitude, i.e. $F_{c}=0.5$. The controlled state trajectory is shown in Fig. 3 (a), while the region around origin is additionally zoomed-in for the sake of visualization. One can see that after the state convergence, in the sliding mode, a stable low-amplitude $\left(\times 10^{-5}\right)$ limit cycle around zero appears due to the by-effects caused by the nonlinear presliding [18] friction. This is not surprising since $F_{c}=0.5 U$, and no explicit presliding friction compensation is performed by the terminal sliding mode control which is only switching between $u= \pm U$. The convergence of both state variables $\left(x_{2}\right.$ is down-scaled by factor 0.1 for the sake of visualization) is shown in Fig. 3 (b) over the progress of frictional perturbation. It can also be side-noted that the feasibility of the Coulomb friction compensation by means of a classical (first-order) sliding mode control with discontinuous control action was experimentally demonstrated, in combination with a time-optimal bang-bang strategy, in a case study presented in [19].

\section{B. Motion system with harmonic perturbation}

As next, we assume an internal (or external) perturbation to be a periodic function of time, modeled by $\xi(t)=$ $0.5 \sin (20 t)$. Note that such disturbing harmonic forces (or 

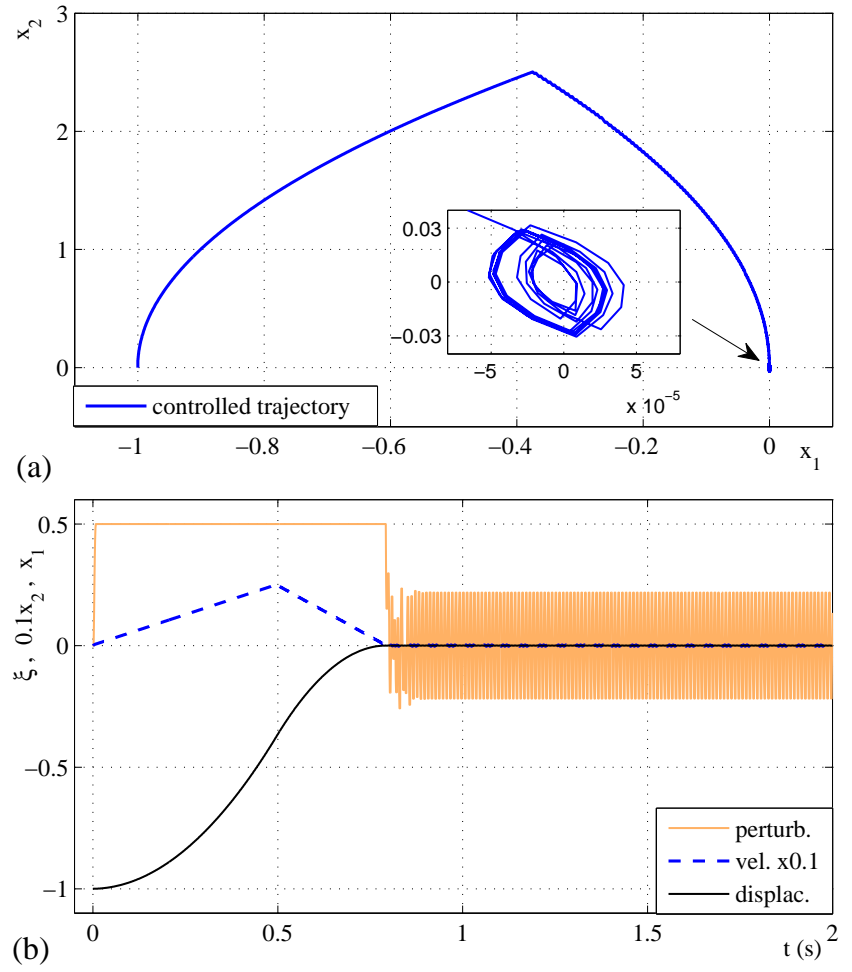

Fig. 3. Phase portrait of state trajectory (a) and convergence of output tracking variable $x_{1}(t)$ and down-scaled $x_{2}(t)$-state over perturbation (b).

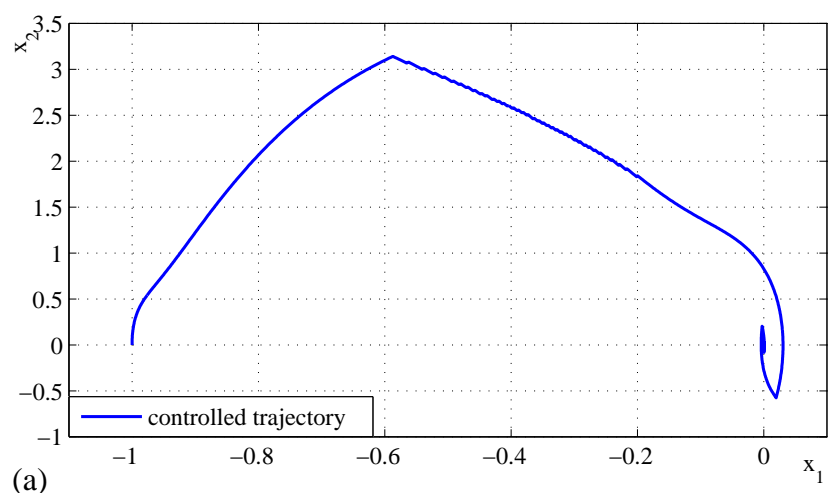

(a)

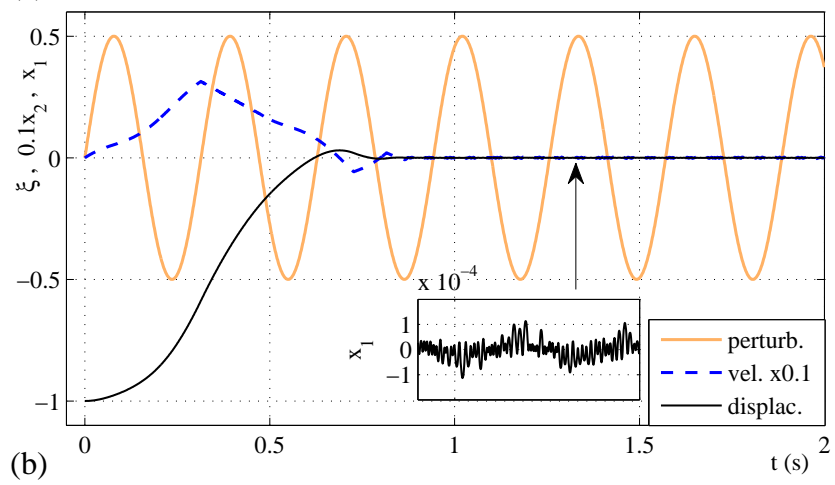

Fig. 4. Phase portrait of state trajectory (a) and convergence of output tracking variable $x_{1}(t)$ and down-scaled $x_{2}(t)$-state over perturbation (b).

torques) may occur in various types of the motors, or more generally actuators, for example see [20]. The sinusoidal amplitude is selected to be the half of the control value, and the angular frequency of $20 \mathrm{rad} / \mathrm{s}$ is assigned for providing a sufficient number of periods during both the transient and steady-state response. The controlled state trajectory and the converged state and perturbation values are shown in Fig. 4. Also the zoomed-in steady-state output error is plotted.

\section{Motion system with random binary perturbation}

Finally, we consider the motion system affected by an external stepwise perturbation which is appearing in form of a random binary signal. The perturbation amplitude is kept at the same level as in both previous examples (cf. Sections $\mathrm{V}-\mathrm{A}$ and $\mathrm{V-B}$, so that the perturbed plant 28 has $\xi(t) \in\{-0.5,0.5\}$ which is a random realization of the binary variable. The controlled state trajectory is shown
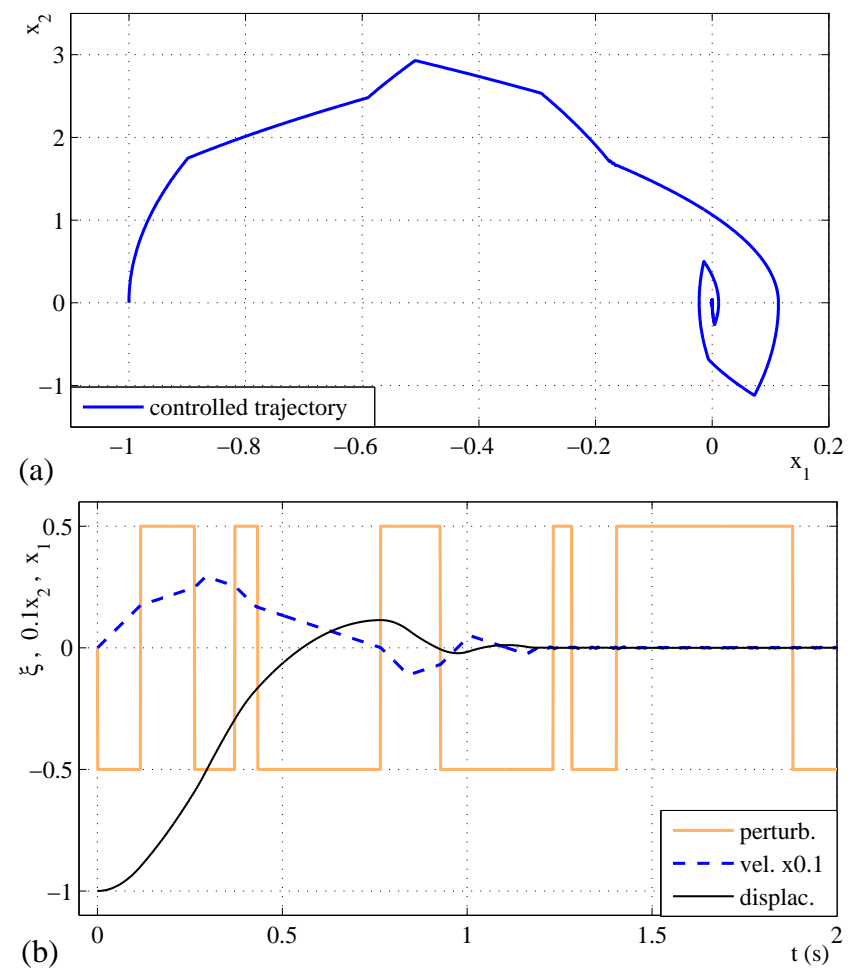

Fig. 5. Phase portrait of state trajectory (a) and convergence of output tracking variable $x_{1}(t)$ and down-scaled $x_{2}(t)$-state over perturbation (b).

in Fig. 5(a), and, alike, in (b) the states convergence and the perturbation value are plotted together as the time series. One can recognize that the motion trajectory is highly perturbed. Yet, both states converge to zero, piecewise in the sliding mode and piecewise in the twisting-like mode. The latter appears transiently each time the binary perturbation changes the sign. It can be stressed that from all three types of the perturbation examples, this one constitutes the 'worst' case which is largely affecting the control system. This is not surprising since the differential changes of the $\xi$-amplitude are of the same magnitude as $U$. The stepwise perturbation $\xi(t)$ does not allow system to continuously staying in the sliding mode and forces it multiple times into the reaching phase. This is directly reflected in the shape of $\left(x_{1}, x_{2}\right)$ 
trajectory, see Fig.5(a). At the same time, one can recognize that the states remain in vicinity to the origin, once reaching it and despite the further stepwise changes of $\xi(t)$, see Fig. 5 (b) for the times $t>1.25 \mathrm{sec}$.

\section{CONCLUDING REMARKS}

This paper described and analyzed the optimal terminal sliding mode control aimed for the second-order motion systems with matched and bounded perturbations. The control scheme uses the time-optimal trajectory of the doubleintegrator dynamics, for which only one dedicated switching provides the maximal possible acceleration and deceleration in presence of the inherent control bounds. The proposed optimal terminal sliding mode has the single free (and rather optional) design parameter $\alpha$, which allows for both, the terminal and twisting control modes. The time-optimal twisting mode on the boundary layer, i.e. for $\alpha=0.5$, is in particular interesting for those motion control applications where a frequent sliding mode switching of the control signal may be undesirable, like in the hydraulic control valves [21]. Here we however abstain from discussions on the pros and cons of the discontinuous and continuous sliding mode controllers, see [22] for details. We focus rather on the analysis and prove of existence and reachability conditions of the proposed optimal terminal sliding mode control. The shown developments and the determined optimal sliding surface are fully inline with the Fuller's problem. It was shown that for a motion dynamics with the given inertial and actuator saturation parameters, there is no other time-optimal surface than (13). All $\alpha>0$ provide a stable state convergence to the origin, while the $\alpha$ values around boundary layer (i.e. $\alpha=0.5$ ) are particularly interesting for the control application. These allow finding a trade-off between a time-optimal convergence with minimal switching and ability to capture multiplicative uncertainties of the inertial and saturation parameters. Three illustrative examples of the bounded internal and external perturbations demonstrated the control efficiency in a numerical setup.

\section{REFERENCES}

[1] V. T. Haimo, "Finite time controllers," SIAM Journal on Control and Optimization, vol. 24, no. 4, pp. 760-770, 1986.

[2] E. Moulay and W. Perruquetti, "Finite-time stability and stabilization: State of the art," in Advances in Variable Structure and Sliding Mode Control. Springer, 2006, pp. 23-41.

[3] V. I. Utkin, Sliding modes in contr. and optim. Springer, 1992.

[4] W. Perruquetti and J.-P. Barbot, Sliding mode control in engineering. CRC press, 2002.

[5] Y. Shtessel, C. Edwards, L. Fridman, and A. Levant, Sliding mode control and observation. Springer, 2014.

[6] M. Zak, "Terminal attractors for addressable memory in neural networks," Physics Letters A, vol. 133, no. 1-2, pp. 18-22, 1988.

[7] S. T. Venkataraman and S. Gulati, "Control of nonlinear systems using terminal sliding modes," in American Control Conference, 1992, pp. 891-893.

[8] Z. Man, A. P. Paplinski, and H. R. Wu, "A robust mimo terminal sliding mode control scheme for rigid robotic manipulators," Transactions on automatic control, vol. 39, no. 12, pp. 2464-2469, 1994.

[9] Y. Feng, X. Yu, and Z. Man, "Non-singular terminal sliding mode control of rigid manipulators," Automatica, vol. 38, no. 12, pp. 21592167, 2002.

[10] A. Fuller, "Relay control systems optimized for various performance criteria," IFAC Proceedings Volumes, vol. 1, no. 1, pp. 520-529, 1960.
[11] F. Dinuzzo and A. Ferrara, "Higher order sliding mode controllers with optimal reaching," IEEE Transactions on Automatic Control, vol. 54, no. 9, pp. 2126-2136, 2009.

[12] A. Bryson, Applied Optimal Control: Optimization, Estimation and Control. CRC Press, 1975.

[13] A. Levant, "Sliding order and sliding accuracy in sliding mode control," International journal of control, vol. 58, no. 6, pp. 1247$1263,1993$.

[14] L. Fridman, J. A. Moreno, B. Bandyopadhyay, S. Kamal, and A. Chalanga, "Continuous nested algorithms: The fifth generation of sliding mode controllers," in Recent advances in sliding modes: From control to intelligent mechatronics. Springer, 2015, pp. 5-35.

[15] T. Sánchez and J. A. Moreno, "Lyapunov functions for twisting and terminal controllers," in 13th International Workshop on Variable Structure Systems (VSS). IEEE, 2014, pp. 1-6.

[16] H. Geering, Optimal control with eng. applic. Springer, 2007.

[17] C. Edwards and S. Spurgeon, Sliding mode control: theory and applications. CRC Press, 1998.

[18] M. Ruderman, "On break-away forces in actuated motion systems with nonlinear friction," Mechatronics, vol. 44, pp. 1-5, 2017.

[19] M. Ruderman and M. Iwasaki, "Hybrid positioning control using timeoptimal feed-forwarding and sliding mode feedback," in France-Japan and Europe-Asia Congress on Mecatronics. IEEE, 2014, pp. 189-194.

[20] M. Ruderman, A. Ruderman, and T. Bertram, "Observer-based compensation of additive periodic torque disturbances in permanent magnet motors," IEEE Transactions on Industrial Informatics, vol. 9, no. 2, pp. 1130-1138, 2013.

[21] M. Ruderman, L. Fridman, and P. Pasolli, "Virtual sensing of load forces in hydraulic actuators using second-and higher-order sliding modes," Control Engineering Practice, vol. 92, p. 104151, 2019.

[22] U. Pérez-Ventura and L. Fridman, "When is it reasonable to implement the discontinuous sliding-mode controllers instead of the continuous ones? frequency domain criteria," International Journal of Robust and Nonlinear Control, vol. 29, no. 3, pp. 810-828, 2019. 